\title{
Laparoscopic excision of a jejunal mesenteric pseudocyst: Report of a case
}

\author{
Aman Gapbarov, ${ }^{1}$ Jülide Sağıroğlu, ${ }^{1}$ Nesrin Gündüz, ${ }^{2}$ \\ Rabia Burçin Girgin, ${ }^{3}$ Gözde Kır, ${ }^{3}$ Orhan Alimoğlu' \\ 'Department of General Surgery, İstanbul Medeniyet University Faculty of Medicine, İstanbul, Turkey \\ ${ }^{2}$ Department of Radiology, İstanbul Medeniyet University Faculty of Medicine, İstanbul, Turkey \\ ${ }^{3}$ Department of Pathology, İstanbul Medeniyet University Faculty of Medicine, İstanbul, Turkey
}

\begin{abstract}
Mesenteric pseudocysts are benign lesions with an unknown etiology. They can present with a moving mass in the abdomen, abdominal pain, or even acute abdomen. Gastrointestinal stromal tumors, mucinous cystadenoma, lymphangioma, endometriosis, mesenteric panniculitis, hydatid cyst, dermoid cyst, and peritoneal cyst should be considered in the differential diagnosis. A 50-year-old female with hypertension and diabetes mellitus presented at the emergency room with intermittent abdominal pain. Contrast-enhanced magnetic resonance imaging revealed a cystic mass $5 \mathrm{~cm}$ in size in the left abdominal region. Laparoscopic exploration was performed and the mass was observed to the right of the ligament of Treitz above the jejunal mesentery. The mass was excised laparoscopically. Analysis of a frozen section indicated benign lesions. The patient was discharged on the second postoperative day without any complications. There were no problems observed at the 6-month follow-up. Mesenteric pseudocysts are usually benign lesions and can be excised laparoscopically.
\end{abstract}

Keywords: Laparoscopic excision; mesenteric; pseudocyst.

\section{Introduction}

Mesenteric pseudocysts are benign lesions that are predicted to form due to lymph node inflammation, lymphatic obstruction, ectopic lymphoid tissue proliferation, congenital diseases, previous pelvic surgery or pelvic inflammatory disease. ${ }^{[1]}$ Approximately $3 \%$ of these lesions follow a malignant course. ${ }^{[2]}$ The predicted mechanism behind mesenteric pseudocysts is lymphatic or purulent material that fills between mesentery sheets as a result of infections or trauma forming a pseudocyst, the wall of which includes necrotic material, fibrin, macrophages and lympho- cytes with no epithelium in the inner lining. ${ }^{[1]}$ In order to avoid confusion with pancreatic pseudocysts, mesenteric pseudocysts are also called non-pancreatic pseudocysts. ${ }^{[3]}$

Intraabdominal pseudocysts are first described in literature by Italian anatomist Benevenni in 1507 in the report of an autopsy performed on an 8-year old boy. ${ }^{[4]}$ Mesenteric pseudocysts are encountered equally in males and females. The frequency does not show a racial difference. They are mostly observed in adults between 40-70, and in children under 10 years of age. ${ }^{[1]}$ Usually, they are incidentally detected, yet can rarely be symptomatic. They can 
present with a mobile mass in the abdomen, abdominal pain, nausea, vomiting, abdominal distention, constipation or diarrhea. Acute abdomen due to rupture, intestinal obstruction or volvulus due to the pseudocysts have also been reported. ${ }^{[1,3,4]}$ In this report, we present a 50 year-old woman with laparoscopically treated symptomatic mesenteric pseudocyst, and discuss the relevant literature.

\section{Case Report}

A 50-year old female patient, who had intermittent abdominal pain for a year, presented to the emergency room for increased intensity of the pain in the last couple of days. Abdominal sonography showed an intraabdominal mass. Contrast-enhanced MRI revealed a mass in the abdominal cavity that is located to the left in the umbilicus level, is $50 \times 43 \mathrm{~mm}$ in the axial plane, appears to be intraperitoneal and has contrast-enhancing walls. There was no solid component or contrast-enhancement within the mass. However, there was an appearance consistent with fat tissue density (Fig. 1). Patient's medical history was significant for hypertension and diabetes mellitus, for which she was under treatment. She had no previous surgeries. Laparoscopic exploration was performed. In the exploration, a 6-cm mass, which was in the close proximity of the ligament of Treitz and superior mesenteric vascular structures and had pseudocyst appearance, was observed (Fig. 2). The mass was located on the superior mesenteric vascular structures and was totally excised. Frozen section was in favor of benign cystic lesion. In histopathological examination, S100 was found to be negative in stromal cells, thus eliminating Schwannoma (Fig. 3). The final report was consistent with inflamed pseudocyst with chronic fibrous walls (Fig. 4). The patient was discharged on the second postoperative day without any complications. There was no complication or recurrence on the $6^{\text {th }}$ month follow-up.

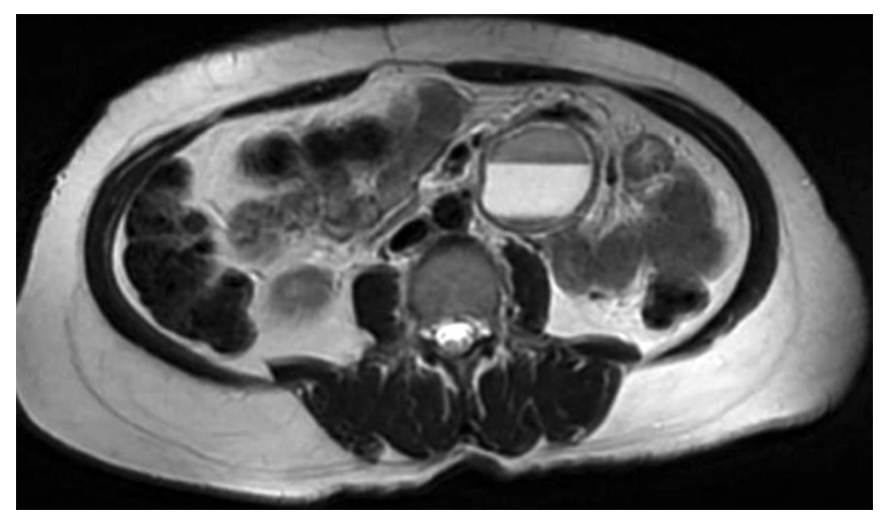

Figure 1. Contrast-enhanced abdominal MRI-axial section.

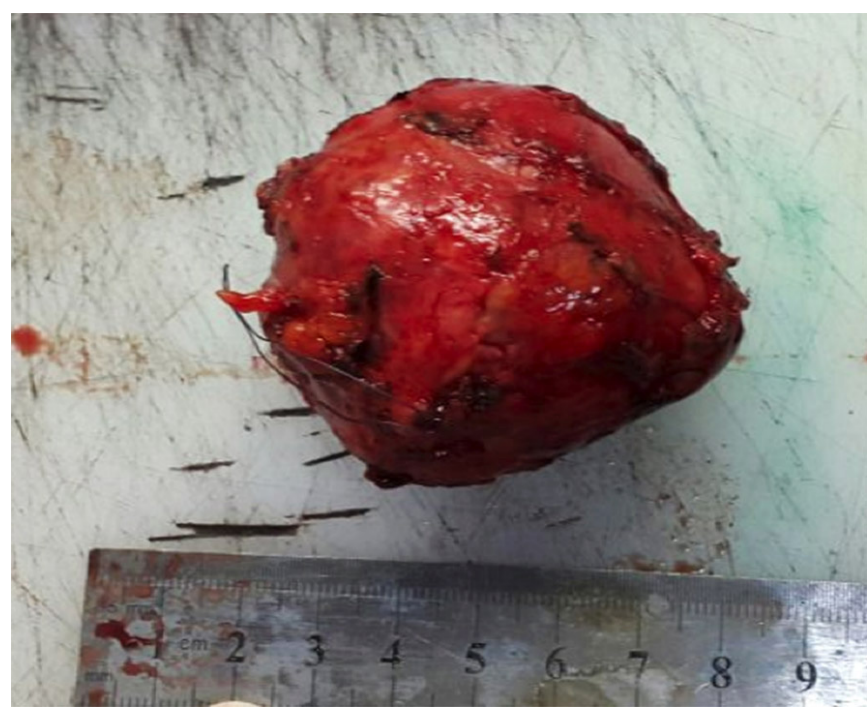

Figure 2. Macroscopic appearance of the mass.

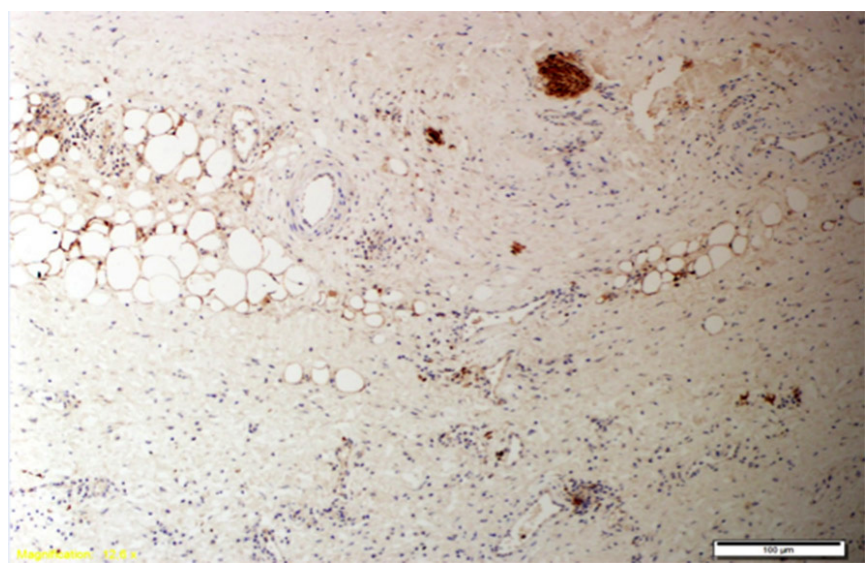

Figure 3. Peripheral tissue used as control was S100 positive whereas the remaining stromal cells were negative.

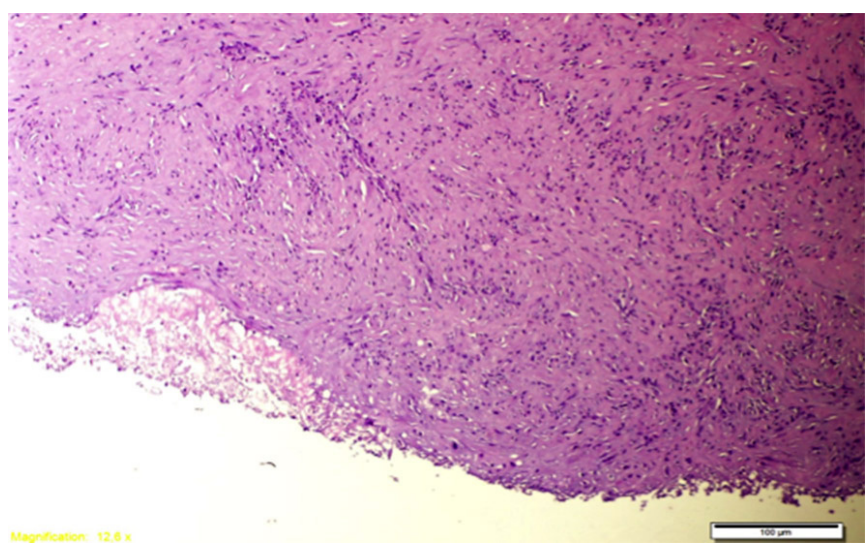

Figure 4. Granulation tissue observed in cyst wall (H\&E, $\mathrm{x} 10)$.

\section{Discussion}

Mesenteric pseudocysts are rarely-encountered benign lesions. According to the literature, the incidence varies between $1 / 100000-1 / 250000 .{ }^{[5]}$ The most common local- 
ization is small intestinal mesentery with $50-80 \%$, as is in our case, followed by colonic mesentery with $15-30 \%$ (right colon more frequently) and retroperitoneal region with $7-20 \%{ }^{[6]}$

The patients are usually asymptomatic and pseudocysts are incidentally detected in imaging performed for other reasons. Complications including infected, bleeding or ruptured pseudocysts may cause clinical symptoms such as abdominal pain, tenderness in the abdomen, distention, nausea and vomiting. Besides, large-sized pseudocysts may cause intestinal obstruction, torsion and bowel ischemia by compression. ${ }^{[7]}$ Our case had no complaints other than intermittent abdominal pain. Ultrasonography, computerized tomography and magnetic resonance imaging can be utilized for diagnosis. ${ }^{[8]}$

Cyst aspiration is not recommended for treatment because the rate of recurrence and complications after aspiration is high. ${ }^{[2,3]}$ The optimal treatment is surgical excision. The laparoscopic approach in mesenteric cyst excision was first performed by Mackenzie et al. in 1993. ${ }^{[9]}$ Laparoscopic surgery is the treatment method that should be primarily considered in these patients. Even though the operation time may be longer with the laparoscopic approach, it is preferred for providing the advantages of minimally invasive surgery including less postoperative pain, shorter hospitalization, quicker recovery and superior esthetic appearance when compared with laparotomy. ${ }^{[6]}$ Our patient was treated using laparoscopic surgery and was discharged without any complications. Prognosis in the postoperative period is quite well and the rate of recurrence is low $(0-13.6 \%)$. ${ }^{[6]}$ There was no recurrence on the 6th month follow-up.

\section{Conclusion}

Mesenteric pseudocysts are benign tumors that are hard to diagnose preoperatively, can only be treated with surgery and should be kept in mind for the differential diagnosis of intraabdominal masses. Laparoscopic surgery should be the primary choice of treatment.

\section{Disclosures}

Informed Consent: Written informed consent was obtained from the patient for the publication of the case report and the accompanying images.

Peer-review: Externally peer-reviewed.

Funding: No institutional or governmental funds have been used for this work.

Conflict of Interest: None declared.

\section{References}

1. Bolívar-Rodríguez MA, Cazarez-Aguilar MA, Luna-Madrid EE, Morgan-Ortiz F. Infected jejunal mesenteric pseudocyst: A case report. Cir Cir 2015;83:334-8. [CrossRef]

2. Kurnicki J, Swiątkiewicz J, Wrzesińska N, Skórski M. Laparoscopic treatment of a huge mesenteric pseudocyst - case report. Wideochir Inne Tech Maloinwazyjne 201 1;6:167-72.

3. Park SE, Jeon TJ, Park JY. Mesenteric pseudocyst of the transverse colon: unusual presentation of more common pathology. BMJ Case Rep 2014;2014.

4. Resta G, Tartarini D, Fabbri N, Bianchini E, Anania G. Laparoscopic resection of a jejunal mesenteric pseudocyst: case report. G Chir 2014;35:279-82.

5. Bhullar JS, Orfanou P. The disappearing abdominal mass: mesenteric pseudocyst. JSLS 2014;18:319-22. [CrossRef]

6. Kumar A, Jakhmola CK, Arora NC, Chauhan SS. Mesenteric cyst in sigmoid mesocolon - A rare location and its laparoscopic excision. Med J Armed Forces India 2015;71:S425-8.

7. Alimoğlu O, Şahin M, Akçakaya A, Karakelleoğlu A, Kaya B. Mesenteric cysts in adults. Turk J Surg 2002;18:278-82.

8. Prakash V, Anushtup D, Roy P, Pandey NK. Pseudomesenteric cyst. Indian J Surg 2009;71:46-7. [CrossRef]

9. Mackenzie DJ, Shapiro SJ, Gordon LA, Ress R. Laparoscopic excision of a mesenteric cyst. J Laparoendosc Surg 1993;3:295-9. [CrossRef] 